\title{
Measurements of MIMO-UWB Indoor Channel
}

\author{
G Tsao, P Iyamu, L Petropoulakis, R Atkinson, I Andonovic, I A Glover \\ Department of Electronic and Electrical Engineering \\ University of Strathclyde \\ Glasgow G1 1XW, UK
}

e-mail: gavin.tsao@strath.ac.uk and osayi.iyamu@gmail.com and \{1.petropoulakis, r.atkinson, i.andonovic, ian.glover.\}@eee.strath.ac.uk

\begin{abstract}
Channel measurements are reported for an emulated indoor MIMO-UWB system. The emulated system represents a ceiling-mounted access-point with two identical UWB, horizontally-polarized, antennas and a portable terminal for desk-top use with between two and four (also identical) horizontally-polarized antennas. The maximum MIMO-UWB channel capacities of the measured channels are calculated and the statistics of these capacities are presented. The channel capacity advantage of MIMO-UWB over SISOUWB is also presented.
\end{abstract}

Keywords-component; UWB, MIMO, channel capacity, indoor, channel measurement.

\section{INTRODUCTION}

Demand for higher wireless data-rates in applications such as multimedia streaming within the home environment continues to increase [1]. Ultra-wideband (UWB) and Multiple-Input Multiple-Output (MIMO) technologies can help in addressing this demand [1].

MIMO systems employ the use of spatial multiplexing, taking advantage of the multipath wireless channel [2].

UWB uses large bandwidth to provide high channel capacity. The large spectral occupancy of UWB signals means their power density must be low, however, and this is enforced by constraining UWB signals to a spectral mask defined by the appropriate radio regulatory authority [3], [4]. Fig. 1 shows an example of a Federal Communications Commission mask used in the USA. Fig. 2 shows a similar mask defined by the Electronic Communication Committee (ECC) for Europe.

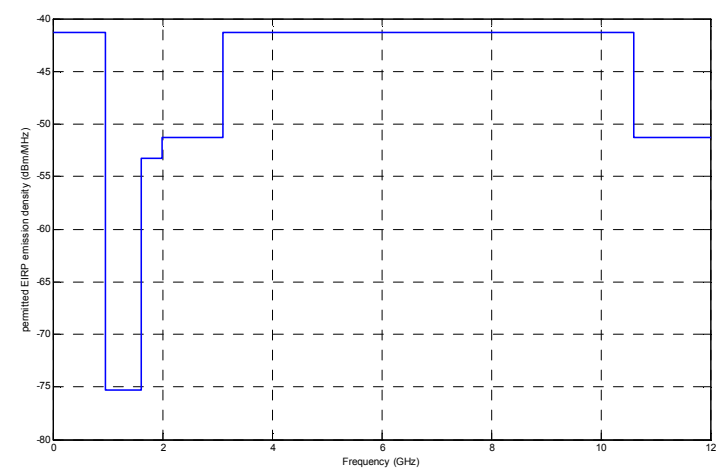

Figure 1. FCC indoor spectrum mask for commercial systems

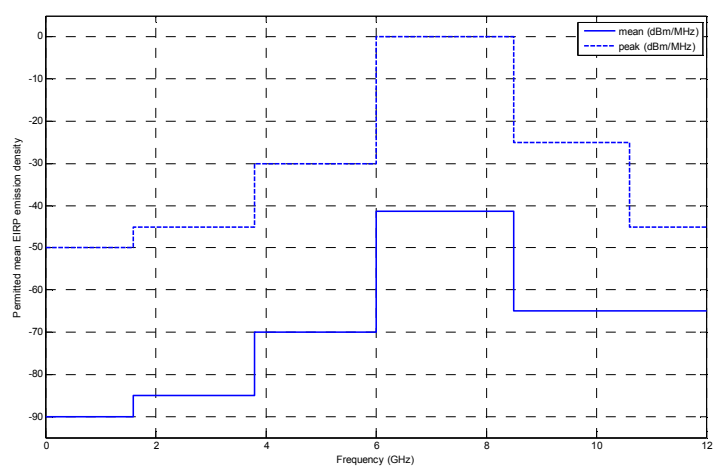

Figure 2. ECC indoor spectrum mask for commercial systems

It is possible to combine both UWB and MIMO technologies to achieve higher data-rates than either technology is capable of supporting alone. As with any wireless communication system, the channel characteristics determine the degree to which the theoretical channel capacity can be realized [5-7].

The experiment described here measures channel characteristics for a $2 \times 2,2 \times 3$, and $2 \times 4$ MIMO-UWB systems in which one 'terminal' is at a fixed, elevated, location and the other 'terminal' is moved systematically over a large horizontal surface (a laboratory bench). The intention is to emulate a ceiling-mounted access point and portable, table-top, device. The maximum theoretical channel capacity is then calculated for these particular, and realistic, channels.

\section{METHODOLOGY}

\section{A. Measurements}

The channel frequency response (or $\mathrm{S}_{21}$ scattering parameter) was measured using a pair of omnidirectional UWB antennas and an Agilent programmable network analyzer (PNA) N5230A [8]. The PNA was set to sweep between $1 \mathrm{GHz}$ and $6 \mathrm{GHz}$ with 16001 sampling points giving a frequency resolution of $312 \mathrm{kHz}$. 20 frequency response measurements were averaged to increase the measurement signal to noise ratio (SNR). The environment was static during these measurements. 


\section{B. UWB antennas}

A pair of identical, biconical, and omni-directional antennas (Fig. 3) was selected for these measurements. The antennas have approximately constant gain (close to $0 \mathrm{dBi}$ ) between $1 \mathrm{GHz}$ and $6 \mathrm{GHz}$ and approximately linear phase [9]. The antennas were connected to the PNA via highquality low-loss flexible coaxial cable [10].

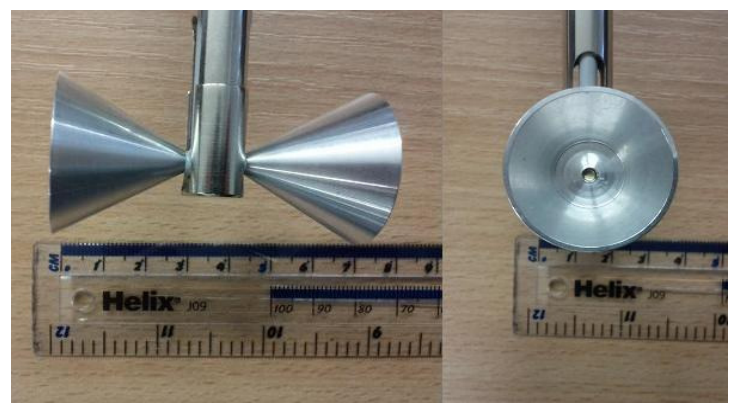

Figure 3. SBA 9119 antenna

\section{Environment}

The (indoor) measurements were performed in the Mobile Communications Laboratory at the University of Strathclyde. The clutter within the environment includes tables (flat laboratory benches), chairs, cupboards, desktop computers and a range of instrumentation (oscilloscopes, spectrum analysers etc).

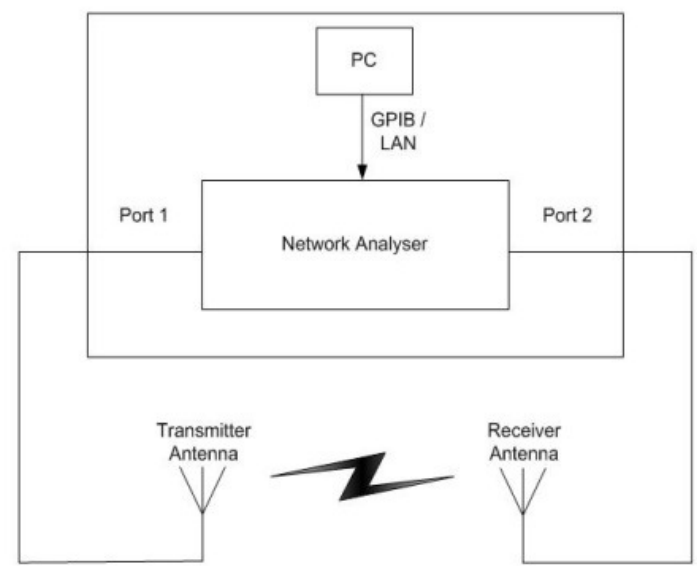

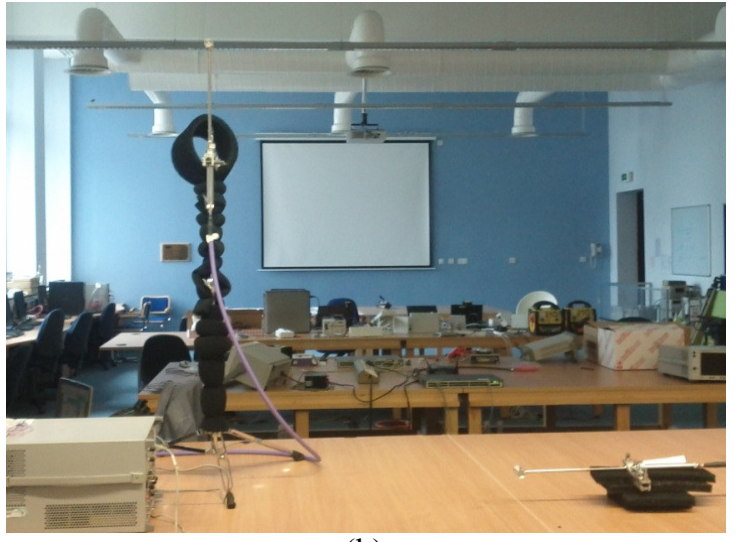

(b)

Figure 4. Measurement setup: (a) schematic diagram, (b) physical realisation

\section{Measurements}

The transmitting antenna emulates a ceiling-mounted access point located at a height of $2.25 \mathrm{~m}$. The measurements were made on two identical large wooden tables placed together. Each table had a rectangular flat surface $1.6 \mathrm{~m} \times$ $1.8 \mathrm{~m}$ at a height of $0.75 \mathrm{~m}$ above the floor. The total surface area available for measurements was thus $1.6 \mathrm{~m} \times 3.6 \mathrm{~m}$. The receiving antenna was placed with its phase centre (assumed to coincide with the symmetry centre of the biconical arms) $10 \mathrm{~cm}$ above the table surface. This was then moved systematically over a square grid $150 \mathrm{~cm} \times 170 \mathrm{~cm}$ in extent with a grid-point separation of $10 \mathrm{~cm}$. Rows and columns of the grid are denoted using numbers and letters respectively as shown in Fig. 5. The transmit antenna was mounted on a metallic stand that was part shrouded with microwave absorber. The stand was located $80 \mathrm{~cm}$ from the long side of the table and $95 \mathrm{~cm}$ from short side. The shortest path length from the transmit antenna (T1) to a receive antenna location was $105 \mathrm{~cm}$. A second selected location for the transmit antenna (T2), was offset from the first location by $10 \mathrm{~cm}$, Fig. 5. Both antennas were oriented with their axes of symmetry horizontal and parallel to measurement point rows. 


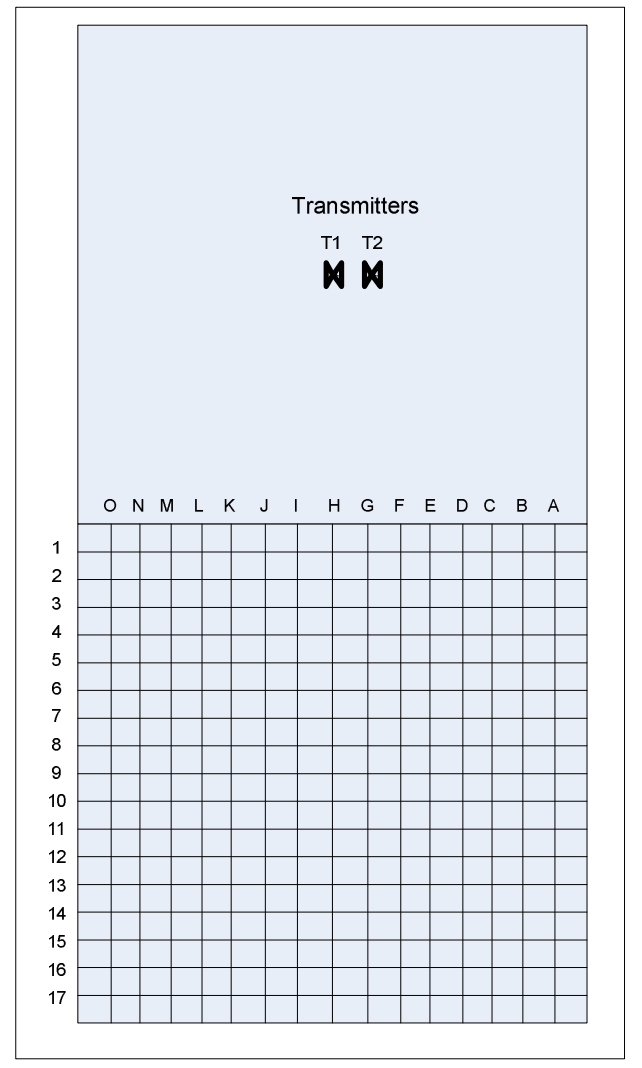

Figure 5. Measurement grid

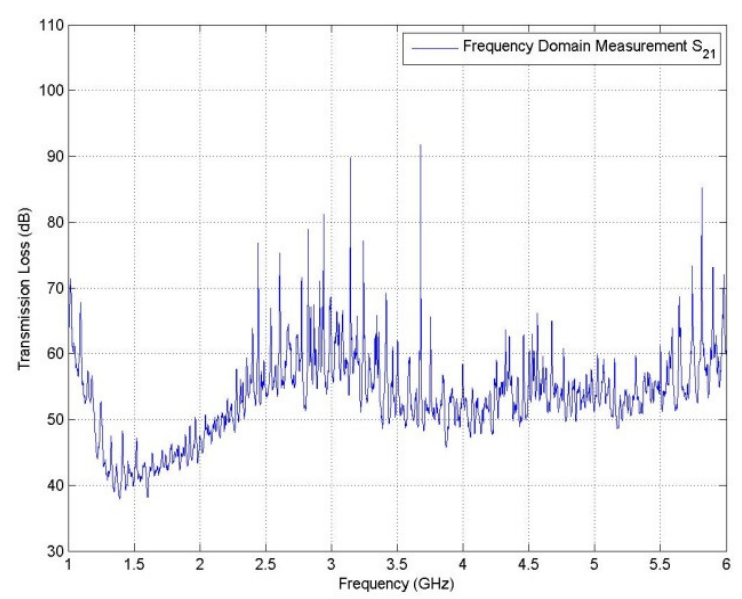

Figure 6. PNA channel measurement

An example channel amplitude response (the amplitude of the measured frequency response) is shown in Fig. 6.

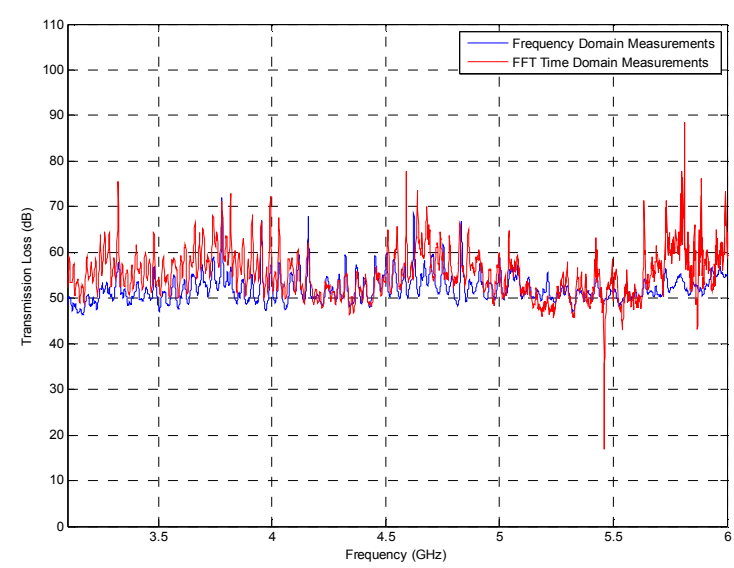

(a)

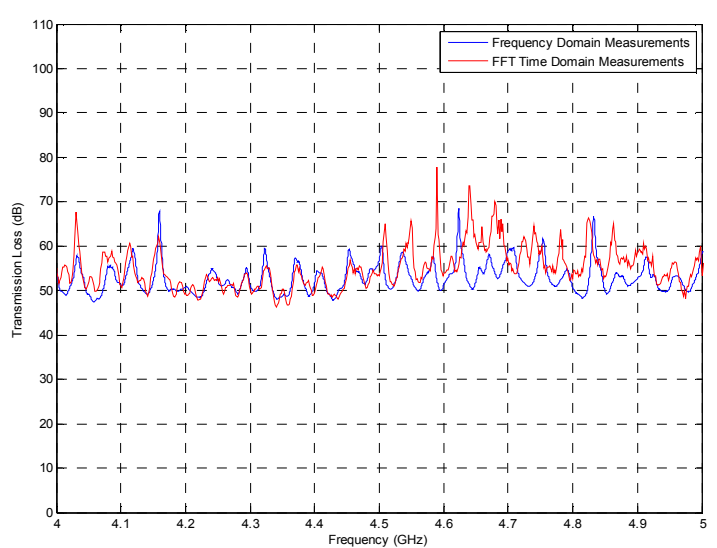

(b)

Figure 7. Comparison between Frequency Domain Measurement and FFT of Time Domain Measurements (a) $3 \mathrm{GHz}$ to $6 \mathrm{GHz}$ (b) $4 \mathrm{GHz}$ to $5 \mathrm{GHz}$.

In order to validate the measurements channel impulse responses were measured for selected measurement geometries using a short ( $80 \mathrm{ps})$ pulse generator and a fast $(9$ $\mathrm{GHz}$ ) digital storage oscilloscope. The fine detail of a channel amplitude response (expressed as transmission loss in $\mathrm{dB}$ ) is compared with the equivalent response, calculated from the Fourier transform of the measured impulse response, in Fig. 7.

The divergence of the two curves above about $4.5 \mathrm{GHz}$ is thought to be due to the PNA calibration which removes the effect of cables and connectors. No such calibration has been undertaken with respect to the impulse response measurement. This close agreement of the fine detail of the two measurements made in different domains with different instruments provides confidence in the measurement procedure.

\section{CHANNEL CAPACITY}

The relationship between received and transmitted frequency domain signals can be described by: 


$$
\mathbf{Y}=\mathbf{h X}+\mathbf{W}
$$

where $\mathbf{Y}$ is the vector of received frequency samples, $\mathbf{X}$ is the vector of transmitted frequency samples, $\mathbf{h}$ is the vector of channel complex gains and $\mathbf{W}$ is the vector of noise.

Shannon's capacity theorem is given by:

$$
C=B \log _{2}\left(1+\frac{E\left(x^{2}\right)}{E\left(w^{2}\right)}\right)
$$

where $C$ is channel capacity, $B$ is channel bandwidth, $x^{2}$ is the instantaneous power in the received signal, $w^{2}$ is the instantaneous power in the receiver noise and $E()$ denotes the expected value.

A single-input single-output (SISO) narrowband channel can be described by:

$$
\mathbf{h}(f)=\sum_{p=1}^{P} a_{p}(f) e^{j \theta_{p}(f)}
$$

where $\mathbf{h}(f)$ is the channel frequency response, $f$ is centre frequency of the narrowband channel, $P$ is the number of propagation paths, $a_{p}$ is the amplitude or strength of the $p^{\text {th }}$ path and $\theta_{p}$ is the phase shift along the $p^{\text {th }}$ path.

A narrowband MIMO channel consists of a matrix of narrowband SISO channels, i.e.:

$$
\mathbf{H}=\left[\begin{array}{ccc}
\mathbf{h}_{1,1} & \cdots & \mathbf{h}_{1, M} \\
\vdots & \ddots & \vdots \\
\mathbf{h}_{N, 1} & \cdots & \mathbf{h}_{N, M}
\end{array}\right]
$$

where $\mathbf{h}_{n, m}$ is the channel link between the $n^{\text {th }}$ receiver and the $m^{\text {th }}$ transmitter of an $N$-receive, $M$-transmit antenna system.

The narrowband MIMO ergodic channel capacity [12] with (spatially) white noise is given by:

$$
C_{M I M O}=B E_{\mathbf{H}}\left\{\log _{2}\left[\operatorname{det}\left(\mathbf{I}_{N}+\frac{1}{\sigma_{w}^{2}} \mathbf{H} \mathbf{R}_{x x} \mathbf{H}^{H}\right)\right]\right\}
$$

where $\mathbf{R}_{x x}$ is the correlation matrix of the transmitted signals, $\mathbf{H}^{H}$ is the hermitian (conjugate transpose) of $\mathbf{H}, \mathbf{I}_{N}$ is the $N \times N$ identity matrix and $\sigma_{w}{ }^{2}$ is the effective Gaussian noise power in the $n^{\text {th }}$ receiver. The narrowband restriction is equivalent to an assumption of flat fading allowing the frequency response of each SISO channel to be represented by a simple complex gain (amplitude and phase shift). The UWB-MIMO channel capacity is the sum of the narrowband MIMO channel capacities, i.e.:

$$
\begin{aligned}
& C_{M I M O-U W B}= \\
& \quad E\left\{\sum_{k=1}^{K} \Delta f \log _{2}\left[\operatorname{det}\left(I_{N}+\frac{1}{\sigma_{w}^{2}} \mathbf{H}_{k} \mathbf{R}_{x x} \mathbf{H}_{k}^{H}\right)\right]\right\}
\end{aligned}
$$

where $K$ is the number of frequency samples and $\Delta f$ is the frequency difference between adjacent samples. If the transmitted signals from the $M$ transmit antennas are uncorrelated then $\mathbf{R}_{x x}$ is given by:

$$
\mathbf{R}_{x x}=\frac{\sigma_{x}^{2} \mathbf{I}_{M}}{M}
$$

where $\mathbf{I}_{M}$ is the $M \times M$ identity matrix and $\sigma_{x}{ }^{2}$ is the total transmitted power.

Equation (6) has been used to calculate the maximum theoretical channel capacity that the measured channels could support for a UWB signal occupying the spectrum between $3.1 \mathrm{GHz}$ and $6 \mathrm{GHz}$. The maximum possible transmitted power is calculated from the FCC indoor spectral mask limiting the transmitted spectral density to -41.3 $\mathrm{dBm} / \mathrm{MHz}$. The noise power, $3.78 \times 10^{-15} \mathrm{~W}$, within the frequency resolution of the measurement has been derived assuming a noise temperature (internal and external) of 878 $\mathrm{K}$ corresponding to an antenna noise temperature of $290 \mathrm{~K}$ and a receiver noise figure of $4.8 \mathrm{~dB}$.

The SISO channel capacity, i.e.:

$$
C_{\text {SISO-UWB }}=E_{\mathbf{H}}\left\{\sum_{k=1}^{K} \Delta f \log _{2}\left(1+\frac{g_{k}^{2} \sigma_{x}^{2}}{\sigma_{w}^{2}}\right)\right\}
$$

has been calculated using identical assumptions where

$$
g^{2}=E\left(|\mathbf{H}|^{2}\right)
$$

The UWB-MIMO capacity advantage has been defined as the ratio of the MIMO-UWB capacity and the SISO-UWB capacity.

The calculations have been carried out for a variety of MIMO configurations. Each configuration assumes two antennas at the emulated access point as illustrated in Fig. 5 and two, three or four antennas at the portable terminal as illustrated in Fig. 8 where $\times$ denotes the location of an antenna on the measurement grid and $\circ$ denotes an empty grid position. Fig. 8(a) shows three 'transverse' configurations for a pair of receive antennas (each configuration with different antenna spacing). Fig. 8(b) shows a 'diagonal' configuration of a pair of antennas. Fig. 8(c) shows three 'transverse' configurations of three antennas. Fig. 8(d) shows four 'L-shaped' configurations of three antennas. Fig. 8(e) shows a 'transverse' configuration of four antennas. Fig. 8(f) shows a 'square' configuration of four antennas.

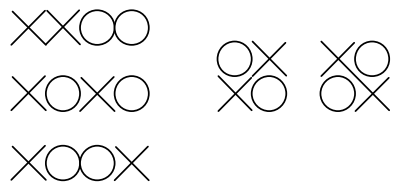

(a)

(b) 


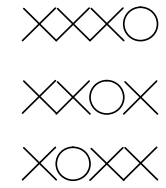

(c)

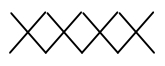

(e)

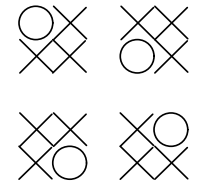

(d)

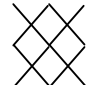

(f)
Figure 8. Emulated antenna configurations at the portable terminal.

The largest spacing between receive antennas is $30 \mathrm{~cm}$. 'Longitude' configurations were also emulated which were identical to 'transverse' configurations but with antenna locations selected from rows of the grid rather than columns.

\section{RESUlts}

Fig. 9 shows the cumulative distribution (CD) of SISO channel capacity for the different receive antenna configurations.

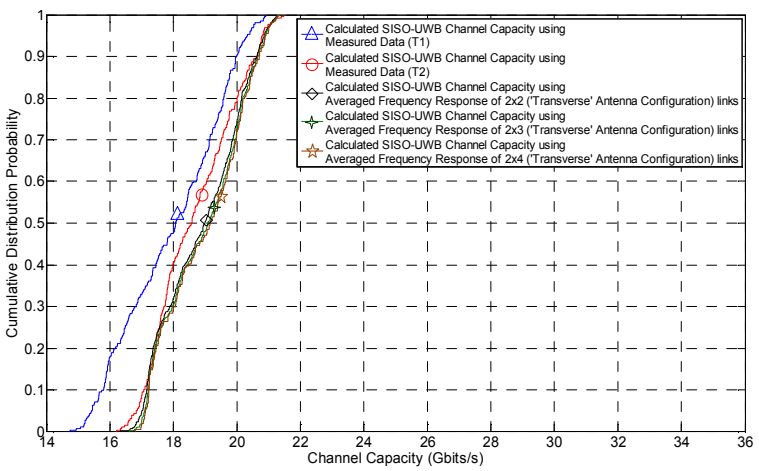

Figure 9. CD of calculated SISO-UWB channel capacities.

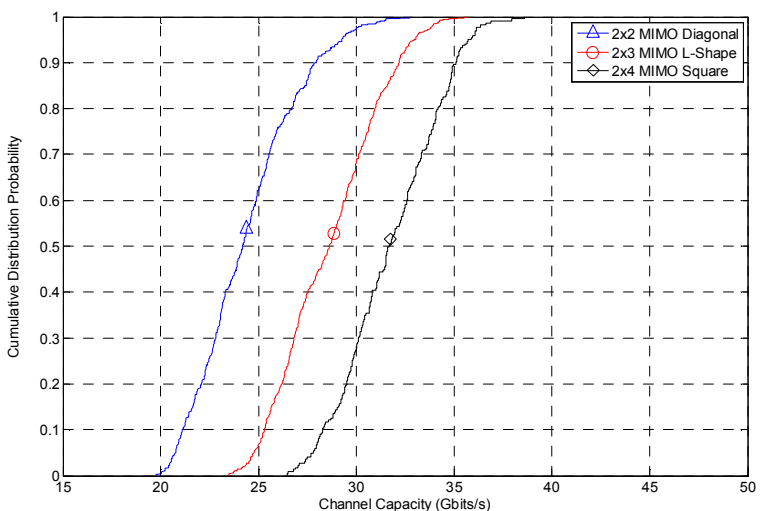

Figure 10. Comparison of MIMO channel capacity for receive antennas in diagonal, $\mathrm{L}$ and square configurations.

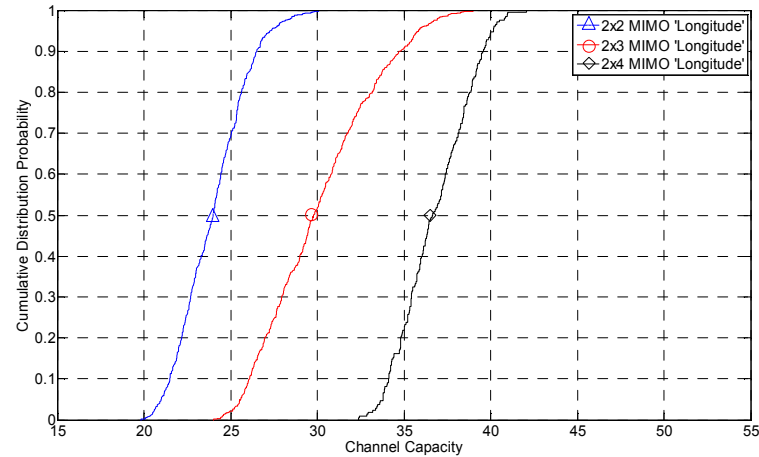

Figure 11. Comparison of MIMO channel capacities for longitudinal receive antenna configurations.

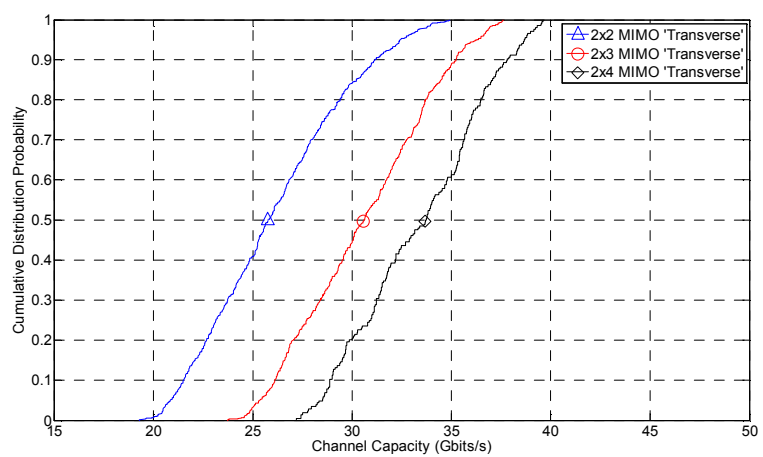

Figure 12. Comparison of MIMO channel capacities for transverse receive antenna configurations.

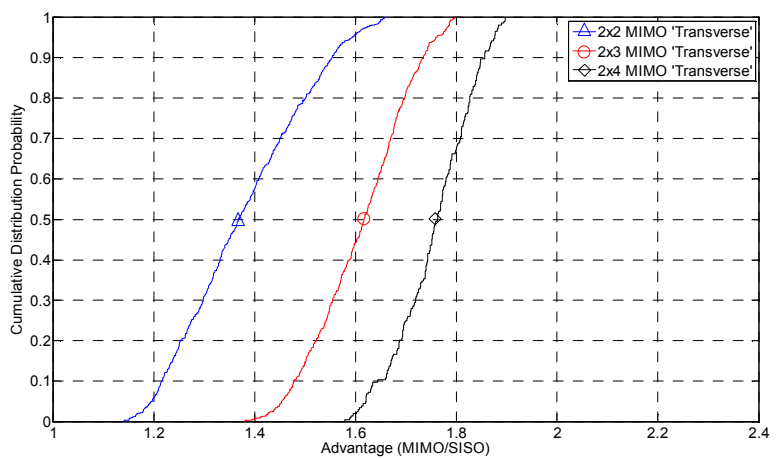

Figure 13. Comparison of MIMO channel advantage for transverse receive antenna configurations.

TABLE I. CHANNEL CAPACITY EXCEEDENCES FOR SEVERAL RECEIVE ANTENNA CONFIGURATIONS.

\begin{tabular}{|l|l|l|l|}
\hline & \multicolumn{3}{|c|}{$2 \times 2$ MIMO (Gbits/s) } \\
\hline & Transverse & Longitude & Diagonal \\
\hline $90 \%$ & 31.14 & 26.49 & 27.94 \\
\hline $50 \%$ & 25.78 & 23.99 & 24.19 \\
\hline $10 \%$ & 21.52 & 21.49 & 21.09 \\
\hline
\end{tabular}




\begin{tabular}{|l|l|l|l|}
\hline & \multicolumn{3}{|c|}{$2 \times 3$ MIMO (Gbits/s) } \\
\hline & Transverse & Longitude & L-Shape \\
\hline $90 \%$ & 35.26 & 31.03 & 32.17 \\
\hline $50 \%$ & 30.59 & 28.24 & 28.63 \\
\hline $10 \%$ & 26.1 & 25.82 & 25.3 \\
\hline
\end{tabular}

\begin{tabular}{|l|l|l|c|}
\hline & \multicolumn{3}{|c|}{$2 \times 4$ MIMO (Gbits/s) } \\
\hline & Transverse & Longitude & Square \\
\hline $90 \%$ & 37.81 & 33.98 & 35.07 \\
\hline $50 \%$ & 33.69 & 31.09 & 31.68 \\
\hline $10 \%$ & 28.88 & 28.71 & 28.27 \\
\hline
\end{tabular}

TABLE II. MIMO ADVANTAGE FOR SEVERAL RECEIVE ANTENNA CONFIGURATION

\begin{tabular}{|l|l|l|l|}
\hline & \multicolumn{3}{|c|}{$2 \times 2$ MIMO Advantage } \\
\hline & Transverse & Longitude & Diagonal \\
\hline $90 \%$ & 1.553 & 1.372 & 1.416 \\
\hline $50 \%$ & 1.369 & 1.266 & 1.274 \\
\hline $10 \%$ & 1.216 & 1.194 & 1.197 \\
\hline
\end{tabular}

\begin{tabular}{|l|l|l|l|}
\hline & \multicolumn{3}{|c|}{$2 \times 3$ MIMO Advantage } \\
\hline & Transverse & Longitude & L-Shape \\
\hline $90 \%$ & 1.735 & 1.581 & 1.607 \\
\hline $50 \%$ & 1.617 & 1.498 & 1.512 \\
\hline $10 \%$ & 1.479 & 1.411 & 1.419 \\
\hline
\end{tabular}

\begin{tabular}{|l|l|l|l|}
\hline & \multicolumn{3}{|c|}{$2 \times 4$ MIMO Advantage } \\
\hline & Transverse & Longitude & Diagonal \\
\hline $90 \%$ & 1.856 & 1.725 & 1.775 \\
\hline $50 \%$ & 1.762 & 1.653 & 1.67 \\
\hline $10 \%$ & 1.645 & 1.557 & 1.577 \\
\hline
\end{tabular}

The theoretical maximum advantage for a $M \times N$ MIMO system over a SISO channel is $\min [M, N]$. All the measurements reported here have two antennas in the emulated access point and at least two antennas in the emulated portable terminal. The maximum theoretical advantage expected for all configurations is therefore 2.0. The maxim and minimum advantages calculated for the measured channels are 1.9 and 1.1 .

\section{CONCLUSION}

The database of measurements acquired provides insight in to the practical MIMO channel capacity advantage that might be expected for an indoor UWB-MIMO system. A range of geometries with two antennas at an emulated ceiling-mounted access point, and between two and four antennas on an emulated portable terminal have been investigated. These early results suggest that the median MIMO advantage realized for such UWB systems is of the order of half the theoretical maximum advantage.

\section{REFERENCES}

[1] T. Kaiser, F. Zheng, and E. Dimitrov, "An Overview of Ultra-Wide-Band Systems With MIMO," Proceedings of the IEEE, vol. 97, no. 2, pp. 285-312, 2009.

[2] A. Sibille, C. Oestges, and Z. Alberto, "MIMO From Theory to Implementation," Elsevier Inc., 2011.

[3] H. Nikookar and R. Prasad, "Introduction to Ultra Wideband for Wireless Communications," Springer, 2009.

[4] L. Yang, G. B. Giannakis, M. Ghavami, and L. Michael, "Ultra-wideband communications: an idea whose time has come," Signal Processing Magazine, IEEE, vol. 21, no. 6, pp. 26-54, 2004.

[5] A. F. Molisch, "MIMO-UWB propagation channels," in Antennas and Propagation (EuCAP), 2010 Proceedings of the Fourth European Conference on, 2010, pp. 1-6.

[6] R. D'Errico, A. Sibille, A. Giorgetti, and M. Chiani, "Antenna diversity in UWB indoor channel," in UltraWideband, 2008. ICUWB 2008. IEEE International Conference on, 2008, vol. 2, pp. 13-16.

[7] A. Sibille, and R. D'Errico, "Multiple antennas effect in UWB spatial multiplexing," in Antennas and Propagation, 2009. 3rd European Conference on, 2009, pp. 249-253.

[8] Aglient Technologies, "Agilent 2-Port PNA-L Microwave Network Analyzer N5230A 300kHz to 6, $13.5 \mathrm{GHz} 10$ MHz to 20, 40, $50 \mathrm{GHz}$ Datasheet," 2011.

[9] Schwarzbeck Mess, "SBA 9119: Microwave Biconical Broadband Antenna 1 ... 6 GHz Manual," 2001.

[10] MegaPhase Labs, EM18, “Low Loss Armored test Cables through $40 \mathrm{GHz}$," 2009. 